\title{
Fuzzy sliding mode controller based on PSO algorithm design
}

\author{
Lihua Li \& Weihua Huang \\ College of Information Science \& Engineering, Wuhan University of Science \& Technology, Wuhan \\ 430081, China
}

Keywords: particle swarm algorithm; the fuzzy sliding mode; parameter optimization; chattering.

\begin{abstract}
For a class of nonlinear systems with uncertainty, a fuzzy sliding mode control method based on particle swarm algorithm, combined with the strong robustness of sliding mode control with fuzzy control is a good approximation capability, use the quasi sliding mode control make the system tracking error within the given boundary layer; Within the boundary layer will replace, switch control with fuzzy control and the supervision function is adopted to control action is smooth. At the same time, to join the supervision and control to diffusion control input and global stability control system has, under the condition of sliding mode existence of controller parameters was optimized by using particle swarm optimization, ensure the sliding mode control of parameter perturbation and external disturbance is strong robustness. Reducing the high frequency chattering of the system, improved the control performance of the controller. The simulation results show the effective-ness of the method.
\end{abstract}

\section{Introduction}

Because of its simple design method of sliding mode variable structure control, the control algorithm easy to implement, from the perspective of theory, the sliding mode can according to the design demand, the sliding mode motion of the system as well as the control object parameters change has nothing to do with the system outside interference, the obvious advantages of sliding mode variable structure control to attract domestic and foreign scholars on the dynamics of different systems, different control requirements for the study of sliding mode variable structure control method, has now formed a relatively independent branch, as a general design method of automatic control system. But sliding mode variable structure of the system parameters perturbation and external disturbance of invariance is a controlled amount of high frequency jitter for high frequency vibration phenomenon, affecting the system steady precision, unmodeled characteristics of excitation system, and even make the system unstable; Secondly because of the system state before reaching the sliding plane do not have the characteristics of sliding mode, thus make the system robustness in the whole dynamic response process is affected. The improvement of traditional sliding mode variable structure control and weaken chattering become the research focus.

To solve above problems, the method of literature (S.S eshagiri, 2002) introduction of boundary layer, both the steady-state error, and weakened the system chattering, but the boundary layer method can only guarantee the system state to sliding surface centered within the boundary layer, only through a narrower to arbitrarily close to the boundary layer sliding mode, not converge to the sliding mode condition. Sun Yibiao (Sun Yibiao 2001) self-learning fuzzy sliding mode control method is proposed, and the controller output is divided into equivalent control and switch control, with fuzzy sliding mode controller to replace the switch control part of sliding mode control, guarantee the continuity of control law, based on the fuzzy basis function of self-learning, to ensure the existence condition and can reach the sliding mode, reducing the conventional sliding mode control to produce high frequency chattering. (Hang C.F.Z 2003) By using the genetic algorithm optimize the switching function, the chattering as the size of the optimization of the important indexes of fitness function, constructs a chattering switching function minimum, showed high in solving nonlinear optimization performance.

In view of the complex control problems, each method has its own limitations, need to combine a variety of methods. The small car inverted pendulum, the author of this paper this kind of nonlinear system, in this paper, a fuzzy sliding mode variable structure control based on particle swarm 
optimization method. The method with strong robustness of sliding mode control with fuzzy control is a good approximation capability, use the quasi sliding mode control make the system tracking error within the given boundary layer, remote control switch control with fuzzy control law, within the boundary layer uses the supervision and control to make the control input is smooth; For better control performance of the controllers, and take the influence of the particle swarm algorithm for fuzzy sliding mode controller system robust performance of several parameters optimization, eliminate the high frequency chattering of sliding mode control system at the same time, the global stability of the system and the steady state accuracy is guaranteed.

\section{System description}

Considering the nonlinear control system with uncertainty

$\left\{\begin{array}{c}\dot{x}_{1}=x_{2} \\ \dot{x}_{2}=x_{3} \\ \vdots \\ \dot{x}_{n}=f(x)+b(x) u+d(t)\end{array}\right.$

Assuming that

$$
f(x, t)=\hat{f}(x, t)+\Delta f(x, t)
$$

In the formula $x=\left[x_{1}, x_{2}, \cdots, x_{n}\right]^{T}$ is the state vector $\mathrm{n}, \mathrm{u}$ is the control of the system input, $\hat{f}(x, t)$ is $f(x, t)$ 's estimates, $\Delta f(x, t)$ is not sure part of the model, $d(t)$ is the external disturbance of the system ,control goal is in the system under the condition of uncertainty and external disturbance, make the system state vector tracking expected trajectory $x_{r}^{T}=\left[x_{r}, \dot{x}_{r}, \ldots, x_{r}^{(n-1)}\right]$,

The error vector is defined as

$$
\begin{aligned}
e^{T} & =\left(e, \dot{e}, \ldots, e^{(n-1)}\right) \\
& =\left(x_{r}-x_{1} \dot{x}_{r}-\dot{x}_{1} \cdots x_{r}^{(n-1)}-x_{1}^{(n-1)}\right) \\
& =\left(e_{1}, e_{2}, \ldots, e_{n}\right)
\end{aligned}
$$

The sliding mode surface function is designed to:

$$
\begin{aligned}
s(X, t) & =\left[\frac{d}{d t}+c\right]^{(n-1)} e \\
& ==e^{(n-1)}+c_{1} e^{(n-2)}+\cdots+c_{n-1}
\end{aligned}
$$

Function of the control law is designed to:

$$
\begin{aligned}
& u(t)=b(X)^{-1}\left(u_{e q}+\Delta u\right) \\
& u_{e q}=-f-\sum_{i=1}^{n-1} c_{i} e_{i+1}+X_{d}^{(n)}(t) \\
& \Delta u=k \operatorname{sgn}(s) \\
& k>(F+D+\eta), \eta>0
\end{aligned}
$$

Among them $\eta>0$.

By (5) - (8) can be proven $s \dot{s} \leq-\eta|s|$, that as long as the type (4) under the condition of constant meet the Hurwitz stable polynomial can prove the stability of sliding mode is gradual and can reach sliding mode condition. According to the defined type (4) shows that the tracking error e and control bandwidth $\lambda$ can be expressed as the relationship lambda $|e| \leq \Phi / \lambda^{n-1}$, $\Phi$ is the boundary layer thickness. Visible, $\lambda$ 's value affect the system steady-state error. The system can achieve stable under 
the premise of the $\lambda$ 's value, the greater the system steady-state error is smaller, the better effect of tracking system will be.

\section{Fuzzy controller design}

Were it not for parameter uncertainties, type (8) of the $\mathrm{k}$ value is too large, easy to produce the chattering. $\mathrm{K}$ value is too small system reach the sliding mode surface time and meet the accessibility of the sliding mode motion would be affected; $\mathrm{K}$ value is too large, easy to cause the chattering. If consider the uncertainty of parameters, parameters of the upper and lower bounds of unknown circumstances, may make the $\mathrm{k}$ value is too big, also not be able to avoid chattering. Ref. (Jenyang Chen 1999) in order to curb this phenomenon, put forward the fuzzy values within the system boundary layer according to the $\mathrm{s}$, blur of $\mathrm{k}$ value adjustment, to realize the switch control regulation of part $\Delta u$, but did not consider $\dot{s}$ change, the uncertainty of parameters may result in instability of the system.

In this paper, fuzzy sliding mode controller is designed, in a neighborhood of the sliding surface $\mathrm{s}$ $=0$ extend a boundary layer $(\Phi=2 \Delta)$, select $\mathrm{s} / \Delta$ and $\dot{s}$ in the boundary layer, $\Delta u^{\prime}$ is the output of fuzzy controller, we use the nonlinear fuzzy language variables are divided into interval method to improve sensitivity of fuzzy controller.

Fuzzy controller input $\mathrm{s} / \Delta, \dot{s}$ and output $\Delta u^{\prime}$ take seven fuzzy sets, with $E_{i}, R_{j}, U_{k}$ respectively. Among them $i, j, k=-3-2,-1,0,1,2,3$; Due to the input $\mathrm{s} / \Delta, \dot{s}$ has seven fuzzy sets, so a total of 49 fuzzy rules,

The fuzzy rules are defined as follows:

If $\mathrm{s} / \Delta$ is $E_{i}$ and $\dot{s}$ is $R_{j}$ then $\Delta u^{\prime}$ is $U_{k}$.

Fuzzy output accurate by gravity method

$$
\Delta u^{\prime}=\frac{\sum_{i=1}^{n} \omega_{i} U_{k}}{\sum_{i=1}^{n} \omega_{i}}
$$

Type in the $\omega_{i}$ weights for the itch rule; In order to improve the sensitivity of the fuzzy controller with a nonlinear partitioning method of fuzzy language variables interval method. Set up A fuzzy variable is $A$, the theory of domain by scaling factor transformation to $[1,1]$ interval, In the view of language domain is divided into five variables $A_{i}(i=[-3,-2,-1,0,1,2,3])$, select triangular membership functions, So the itch a language for type $a_{i}=\frac{i}{3} r^{3-|i|}$ value in the center of the variables, $r \in[0,1]$, when $r=1$, for linear classification, $r$ values obtained too easily lead to fuzzy controller in slow near 0 ; Take the moment to make the value of $\mathrm{r}$ near zero when the fuzzy controller is too sensitive, can't diffusion control input. In this paper, $r$ values range from 0.5-0.9.

A fuzzy controller of language input and output values for PB, PS, Z, NS, NB, membership function is shown in figure $1,2$.

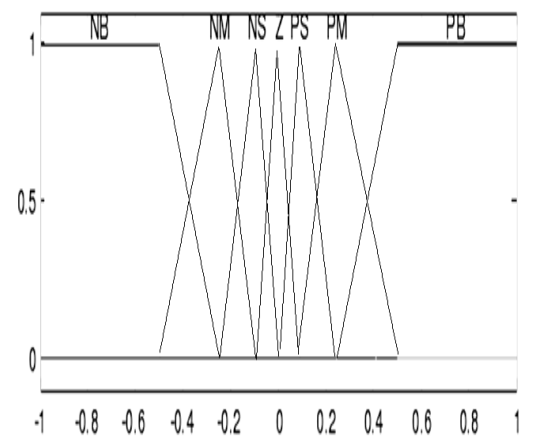

Figure 1 input fuzzy sets $\mathrm{s} / \Delta$ and $\dot{s}$

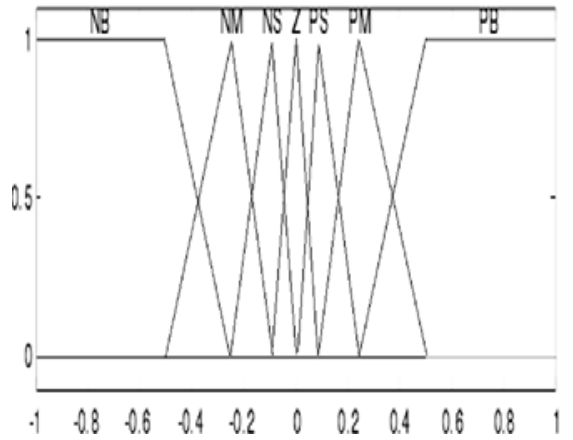

Figure 2 output fuzzy sets $\Delta u^{\prime}$ 
When the system state to reach $|\mathrm{s}|=\mathrm{M}$, In order to weaken the switching control law of the high frequency chattering, introduce a piecewise continuous function $I$ to smooth the $\Delta u^{\prime}$, take $0<I<1$,

$$
\begin{aligned}
& I=\left\{\begin{array}{cc}
0 & |x|<a \\
\frac{|x|-a}{M-a} & a \leq|x| \leq M \\
1 & |x|>M
\end{array}\right. \\
& u_{s}=I^{*} k^{*} \operatorname{sign}(s) \\
& \Delta u^{\prime}=\Delta u+u_{s}
\end{aligned}
$$

In order to guarantee the global stability of the system, when outside the boundary layer, substitute $u_{s}$ for $\Delta \mathrm{u}$ to get smooth control function; Within the boundary layer with $\Delta u^{\prime}$ instead of $\Delta u$ smooth control function. Finally, this paper uses the particle swarm algorithm of parameter to optimize the parameter c, r, and k.

\section{The simulation}

Considering the small car inverted pendulum system, the system for the model equation

$$
\begin{aligned}
& \dot{x}_{1}=x_{2} \\
& \dot{x}_{2}=\frac{g \sin x_{1}-m / x_{2}^{2} \cos x_{1} \sin x_{1} /\left(m_{c}+m\right)}{l\left(4 / 3-m \cos ^{2} x_{1} /\left(m_{c}+m\right)\right)}+\frac{\cos x_{1} /\left(m_{c}+m\right)}{l\left(4 / 3-m \cos ^{2} x_{1} /\left(m_{c}+m\right)\right)} u(t)+d(t)
\end{aligned}
$$

Among them, $x_{1}$ and $x_{2}$ respectively for the system of pendulum Angle and speed, $g=9.8 \mathrm{~m} / \mathrm{s}$; $m_{c}$ is the quality of the car, $m_{c}=1 \mathrm{~kg} ; m$ is the quality of the beam. $u$ Is the input of the controller; System at a given disturbance on a given sine signal curve tracing, the disturbance signal is $d(t)$ $=20 \sin (2 \pi \mathrm{t})$, tracking signal is $y_{d}(t)=\sin (t)$, defined performance indicators are $J=\frac{1}{2} \sum e^{T} e$. The given parameter c range of $[1,20]$, $r$ range of [0.5, 0.9], $k$ range of $[1,30]$, given $\Delta$ value of 1 ; Particle swarm parameters of acceleration factor $\mathrm{c} 1=\mathrm{c} 2=2$, Population size $\mathrm{m}=30$, Reduce the size of the inertia weight $\mathrm{w}$ from 1.1 to 0.2 . Particle fitness function is $F_{\text {fit }}=1 /(J+1)$ after two generations of the optimization, get the optimization of the parameter values: $\mathrm{c}=14.63 ; \mathrm{r}=0.85 ; \mathrm{k}=20.00$.

The simulation results as shown:
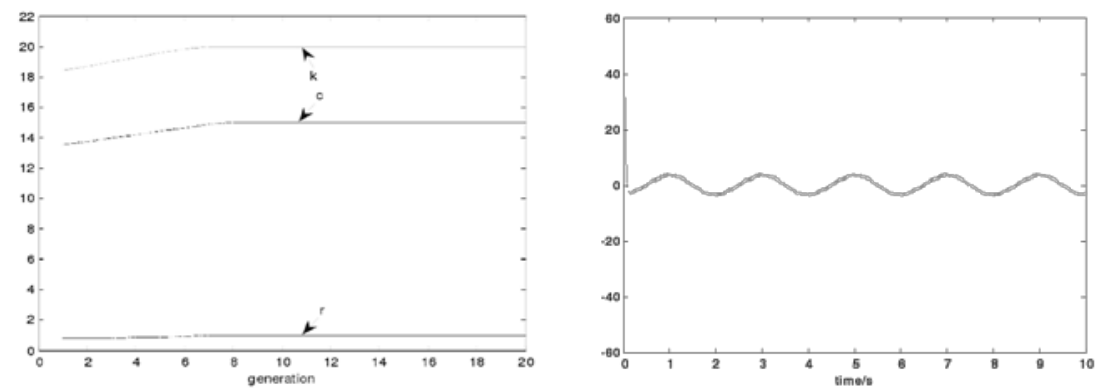

Figure 3 PSO optimization parameter curve Figure 4 control input curve

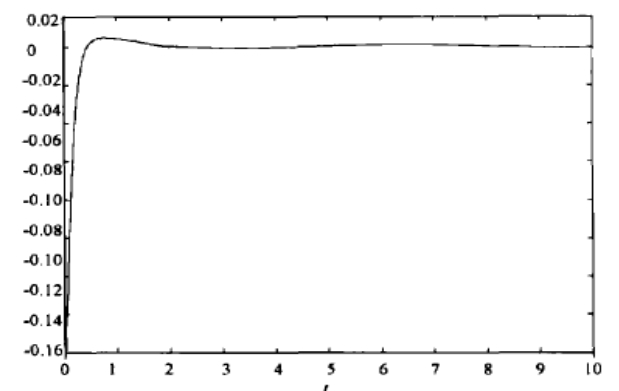

Figure 5 system error curve

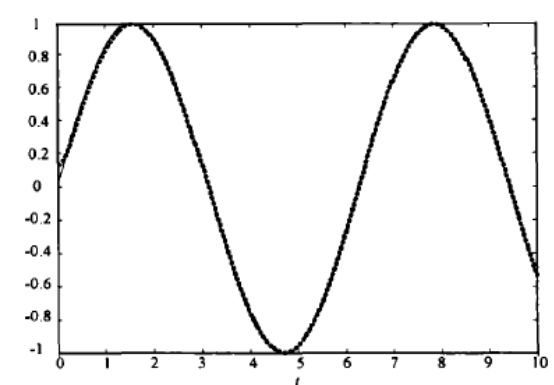

Figure 6 location tracking curve 
The simulation results by above knowable, particle swarm algorithm to optimize the speed faster, in the eighth generation of convergence to the optimal value, eliminate the phenomenon of the high frequency vibration of the system at the same time to improve the performance of the system, and the system can quickly and accurately track the desired trajectory.

\section{Conclusion}

In this paper, we design a fuzzy sliding mode control method based on particle swarm algorithm, combined with the strong robustness of sliding mode control with fuzzy control is a good approximation capability, use the quasi sliding mode control make the system tracking error within the given boundary layer; Within the boundary layer will replace, switch control with fuzzy control and the supervision function is adopted to control action is smooth. At the same time, to join the supervision and control to diffusion control input and global stability control system has, under the condition of sliding mode existence of controller parameters was optimized by using particle swarm optimization, ensure the sliding mode control of parameter perturbation and external disturbance is strong robustness. Which reduces the conventional sliding mode control system in the high frequency chattering problem, and the system to reach steady state a very short time to improve the control performance of the controller, to improve the tracking precision of the system.

\section{References}

[1] Chen Zhimei, zhang well hillock, jian-chao zeng, 1999. Integral fuzzy sliding mode control of the ac servo system [J]. Journal of motor and control, 3 (1): 38-41.

[2] Gao Weibing, 1996. Theory and design method of variable structure control [M]. Beijing: science press.

[3] Hang C.F.Z Y.N.W ang, J.H e, Y.H.L ong 2003. GA NN - integrated Sliding - mode Control System and Its Application in the Printing Press [J]. Control and found \& Applications, 20 (2): 217-222.

[4] Jenyang Chen 1999. Expert SMC - -based Fuzzy Control with based Algorithms [J]. Journal of the Franklin Institute, 336 (4): 589-610.

[5] Lin J F, Chou da n W 2003 induction motor servo drive using sliding - mode controller with based algorithm [J]. J EleetroniePower Systems Researeh, ((2): 93-108.

[6] Liu Jinkun 2005. The sliding mode variable structure control Matlab simulation [M]. Beijing: tsinghua university press.

[7] Lin C K, Wang, s. d, 1999. Fuzzy system identification using an adaptive learning rule with terminal attractors [J]. Fuzzy Sets and system, 101 (3): S343-352.

[8] Piao Ying, 2001. Countries based on fuzzy logic direct adaptive control of a class of nonlinear system [J]. Journal of motor and control, 19 (1): 45 to 50.

[9] S.S eshagiri, H.K.K halil, 2002. On Introducing Integral Action in Sliding Mode Control [C]. Las Vegas, USA: Proceedings of the IEEE Conference on Decision and Control 41 st: 1473-1478.

[10] Sun Yibiao 2001. Ac linear servo system based on fuzzy self-learning sliding mode variable structure control [J]. Journal of electrical engineering technology, (1): 52-56. 